\title{
Exact solution to integrable open multi-species SSEP and macroscopic fluctuation theory
}

\author{
M. Vanicat $d 1$ \\ ${ }^{a}$ LAPTh, CNRS - Université Savoie Mont Blanc. \\ 9 chemin de Bellevue, BP 110, F-74941 Annecy-le-Vieux Cedex, France.
}

\begin{abstract}
We introduce a multi-species generalization of the symmetric simple exclusion process with open boundaries. This model possesses the property of being integrable and appears as physically relevant because the boundary conditions can be interpreted as the interaction with particles reservoirs with fixed densities of each species. The system is driven out-of-equilibrium by these reservoirs. The steady state is analytically computed in a matrix product form. This algebraic structure allows us to obtain exact expressions for the mean particle currents and for the one and two-point correlation functions. An additivity principle is also derived from the matrix ansatz and permits the computation of the large deviation functional of the density profile. We also propose a description of the model in the context of the macroscopic fluctuation theory and we check the consistency with the exact computations from the finite size lattice.
\end{abstract}

LAPTh-060/16

October 2016

\footnotetext{
${ }^{1}$ matthieu.vanicat@lapth.cnrs.fr
} 


\section{Introduction.}

One important challenge of statistical physics is to understand the non-equilibrium stationary states. Such systems, in which the detailed balance is broken, are not governed by a Boltzmann statistics. So far there is no general framework to describe the stationary distribution at the microscopic level. In this perspective, it appears essential to look for exactly solvable stochastic models and to compute analytically their non-equilibrium stationary distribution, in order to put some light on their structure. Finding physically relevant integrable models is a hard task and often requires dealing with the Yang-Baxter equation and with representation theory of quantum groups. The symmetric simple exclusion process (SSEP) is a stochastic model which belongs to this privileged category. It aims to describe a very simple situation where two particle reservoirs at different densities are connected by a pipe. Particles of the highest density reservoir will pour into the lowest density reservoir and the system will display a non vanishing particle current in the stationary regime.

More precisely particles diffuse on a one dimensional lattice connected with the two reservoirs at its extremities. A particle can hop to the left or right neighboring site with equal rate. An hard-core constraint is imposed: there is at most one particle per site. Particles are injected and extracted with asymmetric rates on the boundaries to mimic the coupling with the reservoirs. It turns out that this model is integrable: the Markov matrix governing the stochastic dynamics is identical to the Hamiltonian of the Heisenberg XXX spin chain with open boundaries. The complete spectrum of the Markov matrix has been computed using algebraic Bethe ansatz [34, 5 , and the stationary distribution has been expressed in a matrix product form [22, 19]. The latter has revealed to be a very powerful technique to compute observables in the stationary state and has permitted a rigorous microscopic verification of the Macroscopic Fluctuation Theory (MFT) [23, 24, 20, 19].

There are at least two ways to extend this model. The first is to add an external field in the bulk which induces an asymmetry in the hopping rate of the particles. It leads to the Asymmetric Simple Exclusion Process (ASEP) [10, 13, which also shares the integrability property and displays a rich physical phenomenology with boundary induced phase transitions [31, 22, 38]. The second is to try to extend the model to different species of particles. This is the goal of the present paper. The main difficulty is to tune properly the particles injection and extraction rates in order to maintain the integrability of the model. This requires the solution of the reflection equation, which can be seen as the boundary equivalent of the Yang-Baxter equation. It turns out that at least one solution of this equation has a nice physical interpretation. The exchange rates at the boundaries can be interpreted as an interaction with a particles reservoir which imposes a fixed density for each species.

Let us mention here that the integrable multi-species exclusion processes have received a lot of interest in the last few years. In particular the steady state of the periodic multi-species ASEP was exactly constructed in a matrix product form [2, 3, 28, 33, 36 and its algebraic structure intensively studied [12, 32. The open boundaries case revealed to be more intricate, but some specific example were studied with reflexive boundaries [1], semi permeable boundaries [4, 40] or inhomogeneous hopping rate in the bulk 29]. A large class of integrable boundaries was given in [15] and two-species examples were studied in [14]. The model we propose to study here is simpler than the aforementioned ones because of the symmetric dynamics in the bulk. Nevertheless it displays a non-equilibrium stationary state and seems to be of physical interest. The simple 
matrix product structure of the steady state allows us to push the computations of the physical observables up to the derivation of the large deviation functional of the density profile and allows us to make contact with the MFT.

The plan of the paper reads as follows. In section 2, we present the stochastic dynamics of the model and the associated Markov matrix. Then we construct and prove in section 3 a matrix product expression for the stationary state. The particular case of the thermodynamic equilibrium is also discussed. In section 4, using this algebraic framework, we compute physical quantities such as the mean particles currents and the one and two points correlation functions. Thanks to the matrix ansatz, we formulate in section 5 an additivity principle. It then permits to compute the large deviation functional of the density profile. We propose in section 6 a description of our model in the framework of MFT. We check the consistency with the exact computations from the finite size lattice. Finally in section 7 we recall the integrability framework used to deal with open systems and we give without any proof the expression of the reflection matrices associated to our model.

\section{Presentation of the model.}

\subsection{Dynamical rules.}

We consider a system involving $N$ species of particles which diffuse on a one dimensional lattice comprising $L$ sites. Each site can be in $N$ different states $s=1, \ldots, N$ depending on its occupancy. More precisely, we set $\tau_{i}=s$ if the site at position $i$ carries a particle of species $s$, with $1 \leq s \leq N$. Hence a configuration of the lattice will be denoted by a $L$-uplet $\left(\tau_{1}, \ldots, \tau_{L}\right) \in\{1, \ldots, N\}^{L}$. Let us remark that one of the particle species, for instance species labeled by 1 , may be interpreted as holes on the lattice.

The dynamics is stochastic. During an infinitesimal time $d t$, in the bulk, there is a probability $d t$ that two particles of different species, located on two adjacent sites, exchange their positions. At the left boundary, a particle of species $s^{\prime}$ located on the first site can be replaced by a particle of species $s$ with probability $d t \times \alpha_{s} / a$. In the same way, at the right boundary, a particle of species $s^{\prime}$ located on the last site can be replaced by a particle of species $s$ with probability $d t \times \beta_{s} / b$.

Later on, the parameters $\alpha_{1}, \ldots, \alpha_{N}$ (respectively $\beta_{1}, \ldots, \beta_{N}$ ) will be interpreted as the particle densities at the left (respectively right) reservoirs. We have thus the constraints

$$
\sum_{s=1}^{N} \alpha_{s}=1, \quad \text { and } \quad \sum_{s=1}^{N} \beta_{s}=1 .
$$

The number $a$ (respectively $b$ ) will be seen as the distance between the left reservoir and the first site (respectively the distance between the right reservoir and the last site), the lattice spacing being one in the bulk.

The update rules of the stochastic process described above are summarized in the following table where the rates of the allowed transitions are depicted above the arrows:

\begin{tabular}{|c|c|c|}
\hline Left & Bulk & Right \\
\hline$s^{\prime} \stackrel{\alpha_{s} / a}{\longrightarrow} s$ & $s^{\prime} s \stackrel{1}{\longrightarrow} s s^{\prime}$ & $s^{\prime} \stackrel{\beta_{s} / b}{\longrightarrow} s$ \\
$1 \leq s, s^{\prime} \leq N$ & $1 \leq s, s^{\prime} \leq N$ & $1 \leq s, s^{\prime} \leq N$ \\
\hline
\end{tabular}


Let us stress that the injection and extraction rate of each species at the boundaries are not the most general. The particular model presented here is motivated by the fact that it is integrable (see section 7). It turns out that it has a nice physical interpretation. Taking into account the constraints (1), we are left with $2 \times N$ free parameters. For a generic choice of these parameters, the system will be driven out of equilibrium by the two reservoirs.

Remark. The system will reach, in the long time limit, a thermodynamic equilibrium if and only if the reservoir densities of each species of particle are the same on the left and on the right, namely: $\alpha_{s}=\beta_{s}$, for all $1 \leq s \leq N$. The detailed balance condition is indeed satisfied only in this case.

\subsection{Markov matrix and master equation.}

In this subsection we set up the mathematical formalism needed to write the probability density function of the model and its time evolution (master equation) in a concise vector form. This will be also of great help to compute and express in a simple form the stationary probability density function.

Let us first attach to each site of the lattice a vector space $\mathbb{C}^{N}$ with basis $|1\rangle,|2\rangle, \ldots,|N\rangle$, where $|s\rangle=(\underbrace{0, \ldots, 0}_{s-1}, 1, \underbrace{0, \ldots, 0}_{N-s})^{t}$. The set of all configurations of the lattice is thus embedded in $\underbrace{\mathbb{C}^{N} \otimes \cdots \otimes \mathbb{C}^{N}}_{L}$ with natural basis $\left|\tau_{1}\right\rangle \otimes \cdots \otimes\left|\tau_{L}\right\rangle$, where $\tau_{i}=1,2, \ldots, N$. We denote by $P_{t}\left(\tau_{1}, \ldots, \tau_{L}\right)$ the probability for the system to be in configuration $\left(\tau_{1}, \ldots, \tau_{L}\right)$ at time $t$. These probabilities can be encompassed in a single vector

$$
\left|P_{t}\right\rangle=\left(\begin{array}{c}
P_{t}(1, \ldots, 1,1) \\
P_{t}(1, \ldots, 1,2) \\
\vdots \\
P_{t}(N, \ldots, N, N)
\end{array}\right)=\sum_{1 \leq \tau_{1}, \ldots, \tau_{L} \leq N} P_{t}\left(\tau_{1}, \ldots, \tau_{L}\right)\left|\tau_{1}\right\rangle \otimes \cdots \otimes\left|\tau_{L}\right\rangle .
$$

This allows us to write in a compact form the master equation, governing the time evolution of the probability density

$$
\frac{d\left|P_{t}\right\rangle}{d t}=M\left|P_{t}\right\rangle
$$

where the Markov matrix $M$ is given by

$$
M=B_{1}+\sum_{i=1}^{L-1} m_{i, i+1}+\bar{B}_{L}
$$

The matrices $B, \bar{B}$ and $m$ are the local jump operators. The indices denote the sites, or equivalently the copies of $\mathbb{C}^{N}$, on which the operators act non trivially (they act as the identity in the other copies). The matrix $B$ encodes the dynamics at the left boundary and acts on the first site as

$$
B\left|s^{\prime}\right\rangle=-\frac{1}{a}\left|s^{\prime}\right\rangle+\sum_{1 \leq s \leq N} \frac{\alpha_{s}}{a}|s\rangle, \quad 1 \leq s^{\prime} \leq N,
$$


which leads to the explicit expression

$$
B=\frac{1}{a}\left(\begin{array}{cccccc}
\alpha_{1}-1 & \alpha_{1} & \alpha_{1} & \ldots & \ldots & \alpha_{1} \\
\alpha_{2} & \alpha_{2}-1 & \alpha_{2} & \ldots & \ldots & \alpha_{2} \\
\alpha_{3} & \alpha_{3} & \alpha_{3}-1 & \ldots & \ldots & \alpha_{3} \\
\vdots & \vdots & & \ddots & & \vdots \\
\alpha_{N-1} & \alpha_{N-1} & \ldots & \ldots & \alpha_{N-1}-1 & \alpha_{N-1} \\
\alpha_{N} & \alpha_{N} & \ldots & \ldots & \alpha_{N} & \alpha_{N}-1
\end{array}\right)
$$

In the same way, the matrix $\bar{B}$ encodes the dynamics at the right boundary and acts on the last site as

$$
\bar{B}\left|s^{\prime}\right\rangle=-\frac{1}{b}\left|s^{\prime}\right\rangle+\sum_{1 \leq s \leq N} \frac{\beta_{s}}{b}|s\rangle, \quad 1 \leq s^{\prime} \leq N,
$$

which leads to the explicit expression

$$
\bar{B}=\frac{1}{b}\left(\begin{array}{cccccc}
\beta_{1}-1 & \beta_{1} & \beta_{1} & \ldots & \ldots & \beta_{1} \\
\beta_{2} & \beta_{2}-1 & \beta_{2} & \ldots & \ldots & \beta_{2} \\
\beta_{3} & \beta_{3} & \beta_{3}-1 & \ldots & \ldots & \beta_{3} \\
\vdots & \vdots & & \ddots & & \vdots \\
\beta_{N-1} & \beta_{N-1} & \ldots & \ldots & \beta_{N-1}-1 & \beta_{N-1} \\
\beta_{N} & \beta_{N} & \ldots & \ldots & \beta_{N} & \beta_{N}-1
\end{array}\right) .
$$

Finally the matrix $m$ acts on two adjacent sites and encodes the dynamics in the bulk as

$$
m\left|s^{\prime}\right\rangle \otimes|s\rangle=|s\rangle \otimes\left|s^{\prime}\right\rangle-\left|s^{\prime}\right\rangle \otimes|s\rangle .
$$

It can be expressed as $m=P-1$, where $P$ is the permutation operator, namely $P|v\rangle \otimes|w\rangle=$ $|w\rangle \otimes|v\rangle$ if $|v\rangle,|w\rangle \in \mathbb{C}^{N}$.

Remark. The well-known SSEP model with one species of particles plus holes is recovered from this framework for $N=2$ (one has then to identify species 1 with holes). The present parameters are in this case related to the usual one $\alpha, \beta, \gamma$ and $\delta$ by $\alpha_{1}=\gamma /(\alpha+\gamma), \alpha_{2}=\alpha /(\alpha+\gamma)$, $\beta_{1}=\beta /(\beta+\delta), \beta_{2}=\delta /(\beta+\delta), a=1 /(\alpha+\gamma)$ and $b=1 /(\beta+\delta)$. Note that this corresponds to the change of variable already used to study the one species SSEP, see for instance [19].

\section{Matrix product solution.}

This section is devoted to the construction of the stationary state of the model. More precisely we want to compute the vector $|\mathcal{S}\rangle$ which satisfies the stationary version of the master equation (44), that is $M|\mathcal{S}\rangle=0$. The entries of this vector can be expressed in a matrix product form, that is the probability to observe a configuration $\left(\tau_{1}, \ldots, \tau_{L}\right)$ in the steady state can be written as

$$
\mathcal{S}\left(\tau_{1}, \ldots, \tau_{L}\right)=\frac{1}{Z_{L}}\left\langle\left\langle W\left|X_{\tau_{1}} X_{\tau_{2}} \ldots X_{\tau_{L}}\right| V\right\rangle\right\rangle,
$$


where $Z_{L}=\left\langle\left\langle W\left|C^{L}\right| V\right\rangle\right\rangle$ is a normalisation, so that the entries of $|\mathcal{S}\rangle$ sum to 1 . We have used the notation

$$
C=X_{1}+\cdots+X_{N}
$$

The matrix ansatz was first introduced in the context of exactly solvable out-of-equilibrium models in [22] to construct exactly the steady state of the TASEP. Since then, it has proven to be a very efficient method to solve other models and has been widely used in the literature. The reader can refer to [10] for a review. The connection between the matrix product construction of the stationary state and the integrability of the related model has been pointed out and explained in [16, 37].

\subsection{Algebraic relations.}

For the matrix product state (11) to compute the stationary distribution correctly, the operators $X_{1}, \ldots, X_{N}$ and the boundary vectors $\langle\langle W|$ and $\left.\mid V\rangle\right\rangle$ have to satisfy precise algebraic relations. The root and the meaning of these relations will be exposed in the subsection 3.2. The operators $X_{1}, \ldots, X_{N}$ belong to a Lie algebra 2 . They satisfy the commutation relations

$$
\left[X_{s}, X_{s^{\prime}}\right]=\lambda_{s} X_{s^{\prime}}-\lambda_{s^{\prime}} X_{s}, \quad 1 \leq s, s^{\prime} \leq N
$$

where

$$
\lambda_{s}=\alpha_{s}-\beta_{s}, \quad 1 \leq s \leq N .
$$

Note that the structure constants $\lambda_{s}$ of the Lie algebra (13) can be absorbed after a rescaling of the generators $X_{s} \longrightarrow \lambda_{s} X_{s}$. However, we will not perform this rescaling in the following because it does not simplify the computations of physical quantities.

The action of the operators $X_{s}$ on the left boundary vector $\langle\langle W|$ is given by

$$
\left\langle\langle W|\left(\alpha_{s} C-X_{s}\right)=a \lambda_{s}\langle\langle W|, \quad 1 \leq s \leq N,\right.
$$

where $C$ is defined in (12). Note that these $N$ relations are not all independent (the sum of these equations is trivial), only $N-1$ are necessary. In the same way the action of the operators $X_{s}$ on the right boundary vector $|V\rangle\rangle \mathrm{read}$

$$
\left.\left.\left(\beta_{s} C-X_{s}\right)|V\rangle\right\rangle=-b \lambda_{s}|V\rangle\right\rangle, \quad 1 \leq s \leq N .
$$

Again, only $N-1$ of these equations are independent.

Unfortunately, we were not able to find an explicit representation for the operators $X_{s}$ and the boundary vectors $\langle\langle W|$ and $\mid V\rangle\rangle$. However, we will show that the commutation relations (13) and the relations on the boundary vectors (15) and (16) allow us to compute the currents and correlation functions, see section 4, and to prove an additivity principle, see section 5 .

Remark. Once again the matrix ansatz solution of the usual SSEP with one species of particles and holes can be obtained for $N=2$, by doing the same change of parameters as mentioned in the remark at the end of section 2, and setting $D=X_{2} / \lambda_{2}$ and $E=-X_{1} / \lambda_{1}=X_{1} / \lambda_{2}$. They satisfy $D E-E D=D+E$ and $\langle\langle W|(\alpha E-\gamma D)=\langle\langle W|,(\delta E-\beta D)| V\rangle\rangle=-| V\rangle\rangle$.

\footnotetext{
${ }^{2}$ The Lie algebra (13) is not semi-simple since there is an abelian ideal of rank $N-1$ generated by the elements $\lambda_{1} X_{s}-\lambda_{s} X_{1}$ for $2 \leq s \leq N$. Hence it does not belongs to the well known classification of semi-simple Lie algebras. It could be interesting to study the decomposition into solvable and semi-simple parts of this algebra but this is beyond the scope of this paper.
} 


\subsection{Proof of the matrix product form.}

We now show that the algebraic relations presented above (13), (15) and (16) imply that the matrix product expression (11) gives the stationary state of the model. We need first to define two key vectors

$$
\mathbf{X}=\left(\begin{array}{c}
X_{1} \\
\vdots \\
X_{N}
\end{array}\right) \quad \text { and } \quad \overline{\mathbf{X}}=\left(\begin{array}{c}
\lambda_{1} \\
\vdots \\
\lambda_{N}
\end{array}\right)
$$

They are the building blocks of the algebraic relations presented previously. They allow us to rewrite the vector $|\mathcal{S}\rangle$ in the concise form

$$
|\mathcal{S}\rangle=\frac{1}{Z_{L}}\langle\langle W|\mathbf{X} \otimes \mathbf{X} \otimes \cdots \otimes \mathbf{X}| V\rangle\rangle
$$

Then the commutation relations between the $X_{s}$ (13) can be expressed equivalently as the telescopic relation

$$
m \mathbf{X} \otimes \mathbf{X}=\mathbf{X} \otimes \overline{\mathbf{X}}-\overline{\mathbf{X}} \otimes \mathbf{X} .
$$

In the same way, the equations on the left boundary (15) are equivalent to

$$
\langle\langle W| B \mathbf{X}=\langle\langle W| \overline{\mathbf{X}},
$$

and the equations on the right boundary (16) are equivalent to

$$
\bar{B} \mathbf{X}|V\rangle\rangle=-\overline{\mathbf{X}}|V\rangle\rangle \text {. }
$$

It is known, see for instance [16] for a proof, that relations (19), (20) and (21) are sufficient to ensure that the vector (18) is the stationary state of the Markov matrix (5). Acting with the Markov matrix $M$ on $|\mathcal{S}\rangle$ leads indeed to a telescopic sum.

\subsection{The thermodynamic equilibrium case.}

We already mentioned that the system reaches a thermodynamic equilibrium if and only if $\alpha_{s}=\beta_{s}$ for all $1 \leq s \leq N$. In this case we have $\lambda_{s}=0$ for all $1 \leq s \leq N$, which implies that the operators $X_{s}$ commute one with each other and can be chosen proportional to the identity operator. We hence set $X_{s}:=r_{s}$, with $r_{1}, \ldots, r_{N}$ real numbers. It is straightforward to check that $r_{s}=\alpha_{s}=\beta_{s}$ satisfy the boundary relations (15) and (16).

The steady state is given by

$$
|\mathcal{S}\rangle=\left(\begin{array}{c}
r_{1} \\
\vdots \\
r_{N}
\end{array}\right) \otimes\left(\begin{array}{c}
r_{1} \\
\vdots \\
r_{N}
\end{array}\right) \otimes \cdots \otimes\left(\begin{array}{c}
r_{1} \\
\vdots \\
r_{N}
\end{array}\right) .
$$

This shows that in the thermodynamic equilibrium, the occupation numbers $\tau_{1}, \ldots, \tau_{L}$ are independent and identically distributed random variables. 


\section{Currents and correlation functions.}

The algebraic structure of the stationary state described in section 3 proves very powerful in the computation of physical quantities such as the correlation functions and the particle currents. The first step is to evaluate the normalisation $Z_{L}$.

\subsection{Normalisation}

Assuming that the scalar product of the boundary vectors $\langle\langle W \mid V\rangle\rangle=1$, the normalisation of the steady state defined by $Z_{L}=\left\langle\left\langle W\left|C^{L}\right| V\right\rangle\right\rangle$ is equal to

$$
Z_{L}=\frac{\Gamma(a+b+L)}{\Gamma(a+b)}
$$

where the gamma function satisfies the functional relation $\Gamma(x+1)=x \Gamma(x)$.

Proof. We first remark that because of constraints (1), we have

$$
\sum_{s=1}^{N} \lambda_{s}=\sum_{s=1}^{N} \alpha_{s}-\sum_{s=1}^{N} \beta_{s}=1-1=0
$$

It allows us to compute

$$
\left[X_{s}, C\right]=\sum_{s^{\prime}=1}^{N}\left[X_{s}, X_{s^{\prime}}\right]=\lambda_{s} \sum_{s^{\prime}=1}^{N} X_{s^{\prime}}-X_{s} \sum_{s^{\prime}=1}^{N} \lambda_{s^{\prime}}
$$

and leads to the very useful relation

$$
\left[X_{s}, C\right]=\lambda_{s} C, \quad \text { or equivalently } \quad X_{s} C=C\left(X_{s}+\lambda_{s}\right) .
$$

Using this equality $n$ times we obtain

$$
X_{s} C^{n}=C^{n}\left(X_{s}+n \lambda_{s}\right) .
$$

We are now equipped to compute the normalisation

$$
\begin{aligned}
Z_{L} & =\left\langle\left\langle W\left|C^{L}\right| V\right\rangle\right\rangle=\frac{a \lambda_{1}}{\alpha_{1}} Z_{L-1}+\frac{1}{\alpha_{1}}\left\langle\left\langle W\left|X_{1} C^{L-1}\right| V\right\rangle\right\rangle \\
& =\frac{\lambda_{1}}{\alpha_{1}}(a+L-1) Z_{L-1}+\frac{1}{\alpha_{1}}\left\langle\left\langle W\left|C^{L-1} X_{1}\right| V\right\rangle\right\rangle \\
& =\frac{\lambda_{1}}{\alpha_{1}}(a+b+L-1) Z_{L-1}+\frac{\beta_{1}}{\alpha_{1}} Z_{L} .
\end{aligned}
$$

The first line is obtained thanks to relation (15) for $s=1$. We get the second line through relation (27) for $s=1$ and $n=L-1$. The last equality is established using (16) for $s=1$. Finally (28) can be rearranged and leads to the recursive relation

$$
Z_{L}=(a+b+L-1) Z_{L-1} .
$$

Keeping in mind that $Z_{0}=\langle\langle W \mid V\rangle\rangle=1$, we can solve the previous relation and we obtain (23). 


\subsection{Particle currents.}

The mean stationary current of the particles of species $s$ between site $i$ and $i+1$ is defined by the average algebraic number of particles of species $s$ crossing the bound between sites $i$ and $i+1$ per unit of time:

$$
J_{s}=\frac{\left\langle\left\langle W\left|C^{i-1} X_{s}\left(C-X_{s}\right) C^{L-i-1}\right| V\right\rangle\right\rangle}{Z_{L}}-\frac{\left\langle\left\langle W\left|C^{i-1}\left(C-X_{s}\right) X_{s} C^{L-i-1}\right| V\right\rangle\right\rangle}{Z_{L}} .
$$

Its analytical expression is given by

$$
J_{s}=\frac{\lambda_{s}}{L-1+a+b}
$$

which is independent of the site $i$, as expected from the conservation of the particles number in the bulk.

Proof.

$$
J_{s}=\frac{\left\langle\left\langle W\left|C^{i-1}\left[X_{s}, C-X_{s}\right] C^{L-i-1}\right| V\right\rangle\right\rangle}{Z_{L}}=\frac{\left\langle\left\langle W\left|C^{i-1}\left[X_{s}, C\right] C^{L-i-1}\right| V\right\rangle\right\rangle}{Z_{L}}=\lambda_{s} \frac{Z_{L-1}}{Z_{L}},
$$

where the last equality is obtained thanks to (26). Hence using (23) we get the desired expression (31).

Remark. In the thermodynamic equilibrium case, that is when $\lambda_{s}=0$ for all $s$, all the particle currents vanish, as expected.

\subsection{Correlation functions.}

For a given configuration, we set $\rho_{s}^{(i)}=1$ if there is a particle of species $s$ on the site $i$ and $\rho_{s}^{(i)}=0$ else. The algebraic structure of the steady state, revealed by the matrix product formulation, offers a very efficient framework to compute the equal time multi-points correlation functions in the stationary state $\left\langle\rho_{s_{1}}^{\left(i_{1}\right)} \rho_{s_{2}}^{\left(i_{2}\right)} \ldots \rho_{s_{k}}^{\left(i_{k}\right)}\right\rangle$, where $\langle\cdot\rangle$ stands for the expectation with respect to the stationary measure. We will compute in this subsection only the one and two points correlation functions, which are of particular interest for a physical point of view. In principle closed expressions for the higher order correlation functions can also be derived using the computational techniques presented below.

The one point function $\left\langle\rho_{s}^{(i)}\right\rangle$ represents the mean density of particles of a given species $s$ at a given site $i$. It can be expressed through the matrix product formalism as

$$
\left\langle\rho_{s}^{(i)}\right\rangle=\frac{\left\langle\left\langle W\left|C^{i-1} X_{s} C^{L-i}\right| V\right\rangle\right\rangle}{Z_{L}} .
$$

Using the algebraic structure (see the proof below), it can be reduced to the closed expression

$$
\left\langle\rho_{s}^{(i)}\right\rangle=\frac{(b+L-i) \alpha_{s}+(a+i-1) \beta_{s}}{a+b+L-1} .
$$


Note that the density profile is the linear interpolation between the left reservoir with density $\alpha_{s}$ located at distance $a$ from the first site and the right reservoir with density $\beta_{s}$ located at distance $b$ from the last site. We recover the Fourier law.

The two-point correlation function can also be written in a matrix product form

$$
\left\langle\rho_{s}^{(i)} \rho_{s^{\prime}}^{(j)}\right\rangle=\frac{\left\langle\left\langle W\left|C^{i-1} X_{s} C^{j-i-1} X_{s^{\prime}} C^{L-j}\right| V\right\rangle\right\rangle}{Z_{L}} .
$$

It leads to a factorised expression for the connected two-point function

$$
\begin{aligned}
\left\langle\rho_{s}^{(i)} \rho_{s^{\prime}}^{(j)}\right\rangle_{c} & :=\left\langle\rho_{s}^{(i)} \rho_{s^{\prime}}^{(j)}\right\rangle-\left\langle\rho_{s}^{(i)}\right\rangle\left\langle\rho_{s^{\prime}}^{(j)}\right\rangle \\
& =-\lambda_{s} \lambda_{s^{\prime}} \frac{(a+i-1)(b+L-j)}{(a+b+L-1)^{2}(a+b+L-2)}
\end{aligned}
$$

The formulas (33) and (35) are very similar to the ones derived for the usual one-species SSEP [21, 19] and appear as direct generalisation for the multi-species case.

Proof of (33) and (35). The mean particle density of species $s$ at site $i$ can be computed using the algebraic structure given by the matrix product form

$$
\begin{aligned}
\left\langle\rho_{s}^{(i)}\right\rangle & =\frac{\left\langle\left\langle W\left|C^{i-1} X_{s} C^{L-i}\right| V\right\rangle\right\rangle}{Z_{L}}=(L-i) \lambda_{s} \frac{Z_{L-1}}{Z_{L}}+\frac{\left\langle\left\langle W\left|C^{L-1} X_{s}\right| V\right\rangle\right\rangle}{Z_{L}} \\
& =(b+L-i) \lambda_{s} \frac{Z_{L-1}}{Z_{L}}+\beta_{s}=\frac{(b+L-i) \alpha_{s}+(a+i-1) \beta_{s}}{a+b+L-1} .
\end{aligned}
$$

The second equality is obtained using relation (27). We use then (16) to get the second line of the equation and the last equality is established thanks to expression (23).

For the two-point function, using again (27) and (16), we have

$$
\begin{aligned}
\left\langle\rho_{s}^{(i)} \rho_{s^{\prime}}^{(j)}\right\rangle & =\frac{\left\langle\left\langle W\left|C^{i-1} X_{s} C^{j-i-1} X_{s^{\prime}} C^{L-j}\right| V\right\rangle\right\rangle}{Z_{L}} \\
& =\lambda_{s^{\prime}}(L-j+b) \frac{\left\langle\left\langle W\left|C^{i-1} X_{s} C^{L-i-1}\right| V\right\rangle\right\rangle}{Z_{L}}+\beta_{s^{\prime}}\left\langle\rho_{s}^{(i)}\right\rangle
\end{aligned}
$$

Replacing $L$ by $L-1$ in the expression (33) we obtain

$$
\begin{aligned}
\frac{\left\langle\left\langle W\left|C^{i-1} X_{s} C^{L-i-1}\right| V\right\rangle\right\rangle}{Z_{L}} & =\frac{Z_{L-1}}{Z_{L}} \frac{(b+L-1-i) \alpha_{s}+(a+i-1) \beta_{s}}{a+b+L-2} \\
& =\frac{Z_{L-1}}{Z_{L}}\left(\left\langle\rho_{s}^{(i)}\right\rangle-\lambda_{s} \frac{i-1+a}{(L-1+a+b)(L-2+a+b)}\right)
\end{aligned}
$$

Substituting back in (39) leads to

$$
\left\langle\rho_{s}^{(i)} \rho_{s^{\prime}}^{(j)}\right\rangle=\left\langle\rho_{s}^{(i)}\right\rangle\left\langle\rho_{s^{\prime}}^{(j)}\right\rangle-\lambda_{s} \lambda_{s^{\prime}} \frac{(a+i-1)(b+L-j)}{(a+b+L-1)^{2}(a+b+L-2)},
$$

which concludes the proof. 


\section{Additivity principle and large deviation of the density profile.}

\subsection{Additivity principle from matrix ansatz.}

In order to write an additivity principle, we will need some definitions. We define two row vectors of size $N$, encompassing the particle densities at the two reservoirs

$$
\boldsymbol{\alpha}=\left(\alpha_{1}, \ldots, \alpha_{N}\right) \text { and } \boldsymbol{\beta}=\left(\beta_{1}, \ldots, \beta_{N}\right) .
$$

We need also the vector

$$
\overline{\boldsymbol{\rho}}(u)=(1-u) \boldsymbol{\alpha}+u \boldsymbol{\beta}
$$

which achieves the linear interpolation between $\boldsymbol{\alpha}$ and $\boldsymbol{\beta}$. Remark that we have the equality

$$
\bar{\rho}_{s}\left(\frac{i+a-1}{a+b+L-1}\right)=\left\langle\rho_{s}^{(i)}\right\rangle
$$

where we recall that $\left\langle\rho_{s}^{(i)}\right\rangle$ is the mean particle density of species $s$ at site $i$.

We denote by $\mathcal{S}_{L}\left(\tau_{1}, \ldots, \tau_{L} \mid \boldsymbol{\alpha}, \boldsymbol{\beta}, a, b\right)$ the probability of the configuration $\left(\tau_{1}, \ldots, \tau_{L}\right)$ in the stationary state for a system of size $L$ with boundary parameters $\boldsymbol{\alpha}, \boldsymbol{\beta}, a$ and $b$. Up to now the length of the lattice and the boundary parameters were omitted in the notation $\mathcal{S}\left(\tau_{1}, \ldots, \tau_{L}\right)$ because there was no ambiguity, but this precision will make sense when formulating the additivity principle. The idea of the additivity principle is to express the stationary weights of a system of size $L$ in terms of the stationary weights of the two subsystems of size $L^{\prime}$ and $L^{\prime \prime}$ (with $L=L^{\prime}+L^{\prime \prime}$ ) obtained by cutting the original system in two parts. The results presented in this subsection are heavily inspired by what was done in [20, 19] for the usual SSEP (with one species of particles plus holes).

For the present model, the additivity principle reads

$$
\begin{aligned}
\mathcal{S}_{L}\left(\tau_{1}, \ldots, \tau_{L} \mid \boldsymbol{\alpha}, \boldsymbol{\beta}, a, b\right) & =-\frac{\Gamma\left(a+b+L^{\prime}\right) \Gamma\left(L^{\prime \prime}+1\right)}{\Gamma(a+b+L)} \oint_{u=1} \frac{d u}{2 i \pi} \frac{1}{u^{a+b+L^{\prime}}(1-u)^{L^{\prime \prime}+1}} \\
& \times \mathcal{S}_{L^{\prime}}\left(\tau_{1}, \ldots, \tau_{L^{\prime}} \mid \boldsymbol{\alpha}, \overline{\boldsymbol{\rho}}(u), a, b\right) \mathcal{S}_{L^{\prime \prime}}\left(\tau_{L^{\prime}+1}, \ldots, \tau_{L} \mid \overline{\boldsymbol{\rho}}(u), \boldsymbol{\beta}, 1-b, b\right)
\end{aligned}
$$

This additivity property can be rewritten using the matrix product formalism. Since the algebraic relations (13), (15) and (16) involving the operators $X_{s}$ and the boundary vectors $\langle\langle W|$ and $\left.\mid V\rangle\right\rangle$ depend explicitly on the boundary parameters, we need to introduce some more notations. We denote by $\widetilde{X}_{s}(u),\langle\langle\widetilde{W}(u)|$ and $\left.\mid \widetilde{V}(u)\rangle\right\rangle$ the operators and boundary vectors associated to the system with parameters $\boldsymbol{\alpha}$ and $a$ for the left reservoir and $\overline{\boldsymbol{\rho}}(u)$ and $b$ for the right reservoir. Namely they satisfy (13), (15) and (16) where $\beta_{s}$ has been replaced by $(1-u) \alpha_{s}+u \beta_{s}$ for all $s$ :

$$
\left[\widetilde{X}_{s}(u), \widetilde{X}_{s^{\prime}}(u)\right]=\widetilde{\lambda}_{s}(u) \widetilde{X}_{s^{\prime}}(u)-\widetilde{\lambda}_{s^{\prime}}(u) \widetilde{X}_{s}(u)=u\left(\lambda_{s} \widetilde{X}_{s^{\prime}}(u)-\lambda_{s^{\prime}} \widetilde{X}_{s}(u)\right)
$$

where

$$
\widetilde{\lambda}_{s}(u)=\alpha_{s}-\left[(1-u) \alpha_{s}+u \beta_{s}\right]=u \lambda_{s}
$$

and for the boundaries

$$
\left\langle\langle\widetilde{W}(u)|\left(\alpha_{s} \widetilde{C}(u)-\widetilde{X}_{s}(u)\right)=a u \lambda_{s}\langle\langle\widetilde{W}(u)|,\right.
$$


and

$$
\left.\left.\left(\left[(1-u) \alpha_{s}+u \beta_{s}\right] \widetilde{C}(u)-\widetilde{X}_{s}(u)\right)|\widetilde{V}(u)\rangle\right\rangle=-b u \lambda_{s}|\widetilde{V}(u)\rangle\right\rangle
$$

where

$$
\widetilde{C}(u)=\widetilde{X}_{1}(u)+\cdots+\widetilde{X}_{N}(u) .
$$

In the same way we denote by $\widehat{X}_{s}(u),\langle\langle\widehat{W}(u)|$ and $\left.\mid \widehat{V}(u)\rangle\right\rangle$ the operators and boundary vectors associated to the system with parameters $\overline{\boldsymbol{\rho}}(u)$ and $1-b$ for the left reservoir and $\boldsymbol{\beta}$ and $b$ for the right reservoir. Namely they satisfy (13), (15) and (16) where $\alpha_{s}$ has been replaced by $(1-u) \alpha_{s}+u \beta_{s}$ for all $s$ and $a$ has been replaced by $1-b$ :

$$
\left[\widehat{X}_{s}(u), \widehat{X}_{s^{\prime}}(u)\right]=\widehat{\lambda}_{s}(u) \widehat{X}_{s^{\prime}}(u)-\widehat{\lambda}_{s^{\prime}}(u) \widehat{X}_{s}(u)=(1-u)\left(\lambda_{s} \widehat{X}_{s^{\prime}}(u)-\lambda_{s^{\prime}} \widehat{X}_{s}(u)\right),
$$

where

$$
\widehat{\lambda}_{s}(u)=\left[(1-u) \alpha_{s}+u \beta_{s}\right]-\beta_{s}=(1-u) \lambda_{s},
$$

and for the boundaries

$$
\left\langle\langle\widehat{W}(u)|\left(\left[(1-u) \alpha_{s}+u \beta_{s}\right] \widehat{C}(u)-\widehat{X}_{s}(u)\right)=(1-b)(1-u) \lambda_{s}\langle\langle\widehat{W}(u)|,\right.
$$

and

$$
\left.\left.\left(\beta_{s} \widehat{C}(u)-\widehat{X}_{s}(u)\right)|\widehat{V}(u)\rangle\right\rangle=-b(1-u) \lambda_{s}|\widehat{V}(u)\rangle\right\rangle
$$

where

$$
\widehat{C}(u)=\widehat{X}_{1}(u)+\cdots+\widehat{X}_{N}(u)
$$

We have the formula:

$$
\begin{aligned}
\left\langle\left\langle W\left|X_{\tau_{1}} \ldots X_{\tau_{L}}\right| V\right\rangle\right\rangle & =-\oint_{u=1} \frac{d u}{2 i \pi} \frac{1}{u^{a+b+L^{\prime}}(1-u)^{L^{\prime \prime}+1}} \\
& \left.\times\left\langle\left\langle\widetilde{W}(u)\left|\widetilde{X}_{\tau_{1}}(u) \ldots \widetilde{X}_{\tau_{L^{\prime}}}(u)\right| \widetilde{V}(u)\right\rangle\right\rangle\left\langle\widehat{W}(u)\left|\widehat{X}_{\tau_{L^{\prime}+1}}(u) \ldots \widehat{X}_{\tau_{L}}(u)\right| \widehat{V}(u)\right\rangle\right\rangle .
\end{aligned}
$$

Proof of (57). For $i=1, \ldots, L^{\prime}$ we perform the change of variables

$$
X_{\tau_{i}}=\alpha_{\tau_{i}} C-L_{\tau_{i}} \quad \text { and } \quad \widetilde{X}_{\tau_{i}}(u)=\alpha_{\tau_{i}} \widetilde{C}(u)-\widetilde{L}_{\tau_{i}}(u) .
$$

The new operators $L_{s}$ and $\widetilde{L}_{s}(u)$ behave conveniently on the left boundary

$$
\left\langle\langle W| L_{s}=a \lambda_{s}\left\langle\langle W | \quad \text { and } \quad \left\langle\langle\widetilde{W}(u)| \widetilde{L}_{s}(u)=u a \lambda_{s}\langle\langle\widetilde{W}(u)| .\right.\right.\right.
$$

When we expand the product $X_{\tau_{1}} \ldots X_{\tau_{L^{\prime}}}$ (respectively the product $\tilde{X}_{\tau_{1}}(u) \ldots \widetilde{X}_{\tau_{L^{\prime}}}(u)$ ), we can push the $L_{s}$ (respectively the $\widetilde{L}_{s}(u)$ ) to the left through the $C^{\prime}$ 's (respectively the $\widetilde{C}(u)$ 's) using the relation $\left[L_{s}, C\right]=-\lambda_{s} C$ (respectively the relation $\left[\widetilde{L}_{s}(u), \widetilde{C}(u)\right]=-u \lambda_{s} \widetilde{C}(u)$ ). At the end the expansion of $X_{\tau_{1}} \ldots X_{\tau_{L^{\prime}}}$ involve monomials of the form $\lambda_{s_{1}} \ldots \lambda_{s_{k}} L_{s_{k+1}} \ldots L_{s_{n^{\prime}}} C^{L^{\prime}-n^{\prime}}$. The expansion of the product $\widetilde{X}_{\tau_{1}}(u) \ldots \widetilde{X}_{\tau_{L^{\prime}}}(u)$ is exactly the same but with the previous monomial replaced by $u^{k} \lambda_{s_{1}} \ldots \lambda_{s_{k}} \widetilde{L}_{s_{k+1}}(u) \ldots \widetilde{L}_{s_{n^{\prime}}}(u) \widetilde{C}(u)^{L^{\prime}-n^{\prime}}$.

In the same way for $i=L^{\prime}+1, \ldots, L^{\prime}+L^{\prime \prime}$ we perform the change of variables

$$
X_{\tau_{i}}=\beta_{\tau_{i}} C-R_{\tau_{i}} \text { and } \quad \widehat{X}_{\tau_{i}}(u)=\beta_{\tau_{i}} \widehat{C}(u)-\widehat{R}_{\tau_{i}}(u) .
$$


The new operators $R_{s}$ and $\widehat{R}_{s}(u)$ behave conveniently on the right boundary

$$
\left.\left.\left.\left.R_{s}|V\rangle\right\rangle=-b \lambda_{s}|V\rangle\right\rangle \quad \text { and } \quad \widehat{R}_{s}(u)|\widehat{V}(u)\rangle\right\rangle=-(1-u) b \lambda_{s}|\widehat{V}(u)\rangle\right\rangle .
$$

Following the same idea as previously, the expansion of $X_{\tau_{L^{\prime}+1}} \ldots X_{\tau_{L}}$ involve monomials of the form $\lambda_{s_{1}} \ldots \lambda_{s_{k}} C^{L^{\prime \prime}-n^{\prime \prime}} R_{s_{k+1}} \ldots R_{s_{n^{\prime \prime}}}$. The expansion of the product $\widetilde{X}_{\tau_{L^{\prime}+1}}(u) \ldots \widetilde{X}_{\tau_{L}}(u)$ is exactly the same but with the previous monomial replaced by $(1-u)^{k} \lambda_{s_{1}} \ldots \lambda_{s_{k}} \widehat{C}(u)^{L^{\prime \prime}-n^{\prime \prime}} \widehat{R}_{s_{k+1}}(u) \ldots \widehat{R}_{s_{n^{\prime \prime}}}(u)$.

Putting all these expansions together, we see that finally it remains to prove

$$
\begin{aligned}
\left\langle\left\langle W\left|C^{L^{\prime}+L^{\prime \prime}-n^{\prime}-n^{\prime \prime}}\right| V\right\rangle\right\rangle & =-\oint_{u=1} \frac{d u}{2 i \pi} \frac{1}{u^{a+b+L^{\prime}-n^{\prime}}(1-u)^{1+L^{\prime \prime}-n^{\prime \prime}}} \\
& \left.\times\left\langle\left\langle\widetilde{W}(u)\left|\widetilde{C}(u)^{L^{\prime}-n^{\prime}}\right| \widetilde{V}(u)\right\rangle\right\rangle\left\langle\widehat{W}(u)\left|\widehat{C}(u)^{L^{\prime \prime}-n^{\prime \prime}}\right| \widehat{V}(u)\right\rangle\right\rangle .
\end{aligned}
$$

This is established using result (23) and the fact that

$$
\begin{aligned}
\oint_{u=1} \frac{d u}{2 i \pi} \frac{1}{u^{a+b+L^{\prime}-n^{\prime}}(1-u)^{1+L^{\prime \prime}-n^{\prime \prime}}} & =-\left.\frac{(-1)^{L^{\prime \prime}-n^{\prime \prime}}}{\left(L^{\prime \prime}-n^{\prime \prime}\right) !} \frac{d^{L^{\prime \prime}-n^{\prime \prime}}}{d u^{L^{\prime \prime}-n^{\prime \prime}}} \frac{1}{u^{a+b+L^{\prime}-n^{\prime}}}\right|_{u=1} \\
& =-\frac{\Gamma\left(a+b+L^{\prime}+L^{\prime \prime}-n^{\prime}-n^{\prime \prime}\right)}{\Gamma\left(a+b+L^{\prime}-n^{\prime}\right) \Gamma\left(1+L^{\prime \prime}-n^{\prime \prime}\right)} .
\end{aligned}
$$

\subsection{Large deviation of the density profile.}

We are interested in evaluating the probability of observing in the stationary state a given density profile in the limit of large system size $L$. In order to formalize the problem, we split the full system which contains $L=n l$ sites into $n$ subsystems (called "boxes" below) containing $l$ sites each, see figure 1.

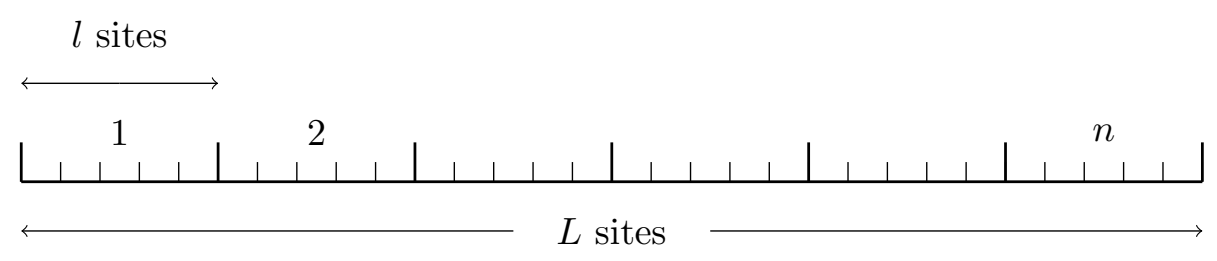

Figure 1: The system of length $L$ is divided into $n$ boxes of length $l$.

We denote by $P_{L}\left(\left\{\boldsymbol{\rho}^{\{1\}}, \boldsymbol{\rho}^{\{2\}}, \ldots, \boldsymbol{\rho}^{\{n\}}\right\} \mid \boldsymbol{\alpha}, \boldsymbol{\beta}\right)$ the probability to find $\rho_{s}^{\{k\}} \times l$ particles 3 of species $s$ in the box $k$ for $s=1, \ldots, N$ and $k=1, \ldots, n$. The row vector $\rho^{\{k\}}$ encompasses the particles densities of each species in the box $k$ :

$$
\rho^{\{k\}}=\left(\rho_{1}^{\{k\}}, \ldots, \rho_{N}^{\{k\}}\right) .
$$

\footnotetext{
${ }^{3}$ the notation $\rho_{s}^{\{k\}}$ (which stands for the mean number of particles of species $s$ in the box $k$ ) should not be confused with the occupation number $\rho_{s}^{(i)}$ defined in subsection 4.3 (which is equal to 1 if there is a particle of species $s$ at site $i$ and 0 else). The link between these variables is $\rho_{s}^{\{k\}}=\frac{1}{l} \sum_{i=k l+1}^{(k+1) l} \rho_{s}^{(i)}$.
} 
Note that we have $\rho_{1}^{\{k\}}+\cdots+\rho_{N}^{\{k\}}=1$, for all $1 \leq k \leq n$.

For $L$ large we expect the probability $P_{L}\left(\left\{\boldsymbol{\rho}^{\{1\}}, \boldsymbol{\rho}^{\{2\}}, \ldots, \boldsymbol{\rho}^{\{n\}}\right\} \mid \boldsymbol{\alpha}, \boldsymbol{\beta}\right)$ to follow a large deviation principle

$$
P_{L}\left(\left\{\boldsymbol{\rho}^{\{1\}}, \boldsymbol{\rho}^{\{2\}}, \ldots, \boldsymbol{\rho}^{\{n\}}\right\} \mid \boldsymbol{\alpha}, \boldsymbol{\beta}\right) \sim \exp \left(-L \mathcal{F}_{n}\left(\left\{\boldsymbol{\rho}^{\{1\}}, \boldsymbol{\rho}^{\{2\}}, \ldots, \boldsymbol{\rho}^{\{n\}}\right\} \mid \boldsymbol{\alpha}, \boldsymbol{\beta}\right)\right) .
$$

In the limit where the number of boxes $n \gg 1$ and the size of each box $l \gg 1$, we can define a continuous coordinate $x$ such that $k=x L$ and a vector $\boldsymbol{\rho}(x)=\boldsymbol{\rho}^{\{k\}}$. We obtain in this case a large deviation functional $\mathcal{F}(\{\boldsymbol{\rho}(x)\} \mid \boldsymbol{\alpha}, \boldsymbol{\beta})$

$$
P_{L}(\{\boldsymbol{\rho}(x)\} \mid \boldsymbol{\alpha}, \boldsymbol{\beta}) \sim \exp (-L \mathcal{F}(\{\boldsymbol{\rho}(x)\} \mid \boldsymbol{\alpha}, \boldsymbol{\beta})) .
$$

In the particular case of the thermodynamic equilibrium, i.e. when $\boldsymbol{\alpha}=\boldsymbol{\beta}:=\boldsymbol{r}=\left(r_{1}, \ldots, r_{N}\right)$, see (22), the large deviation functional is given by

$$
\mathcal{F}(\{\boldsymbol{\rho}(x)\} \mid \boldsymbol{r}, \boldsymbol{r})=\int_{0}^{1} d x B(\boldsymbol{\rho}(x), \boldsymbol{r}),
$$

where

$$
B(\boldsymbol{\rho}, \boldsymbol{r})=\sum_{s=1}^{N} \rho_{s} \ln \left(\frac{\rho_{s}}{r_{s}}\right)
$$

We recall that $r_{1}+\cdots+r_{N}=1$ and $\rho_{1}(x)+\cdots+\rho_{N}(x)=1$ for all $x$. Remark that $B(\boldsymbol{\rho}(x), \boldsymbol{r})$ is nothing else but the Kullback-Leibler divergence between the two discrete measure $\boldsymbol{\rho}(x)$ and $\boldsymbol{r}$.

Proof of (68). In the thermodynamic equilibrium case the stationary distribution is given by (22). Hence we can easily evaluate

$$
P_{L}\left(\left\{\boldsymbol{\rho}^{\{1\}}, \boldsymbol{\rho}^{\{2\}}, \ldots, \boldsymbol{\rho}^{\{n\}}\right\} \mid \boldsymbol{r}, \boldsymbol{r}\right)=\prod_{k=1}^{n} \frac{l !}{\left(l \rho_{1}^{\{k\}}\right) ! \ldots\left(l \rho_{N}^{\{k\}}\right) !} r_{1}^{l \rho_{1}^{\{k\}}} \ldots r_{N}^{l \rho_{N}^{\{k\}}} .
$$

Then using the Stirling formula we obtain

$$
\lim _{l \rightarrow \infty}-\frac{1}{L} \ln P_{L}\left(\left\{\boldsymbol{\rho}^{\{1\}}, \boldsymbol{\rho}^{\{2\}}, \ldots, \boldsymbol{\rho}^{\{n\}}\right\} \mid \boldsymbol{r}, \boldsymbol{r}\right)=\frac{1}{n} \sum_{k=1}^{n} \sum_{s=1}^{N} \rho_{s}^{\{k\}} \ln \left(\frac{\rho_{s}^{\{k\}}}{r_{s}}\right) .
$$

The limit of large $n$ thus gives

$$
\lim _{n \rightarrow \infty} \lim _{l \rightarrow \infty}-\frac{1}{L} \ln P_{L}\left(\left\{\boldsymbol{\rho}^{\{1\}}, \boldsymbol{\rho}^{\{2\}}, \ldots, \boldsymbol{\rho}^{\{n\}}\right\} \mid \boldsymbol{r}, \boldsymbol{r}\right)=\int_{0}^{1} d x \sum_{s=1}^{N} \rho_{s}(x) \ln \left(\frac{\rho_{s}(x)}{r_{s}}\right),
$$

which yields the desired result.

The non-equilibrium case $\boldsymbol{\alpha} \neq \boldsymbol{\beta}$ is more involved:

$$
\mathcal{F}(\{\boldsymbol{\rho}(x)\} \mid \boldsymbol{\alpha}, \boldsymbol{\beta})=\int_{0}^{1} d x\left[B(\boldsymbol{\rho}(x), \overline{\boldsymbol{\rho}}(u(x)))+\ln u^{\prime}(x)\right],
$$


where $u$ is the monotonic solution of the differential equation

$$
\frac{u^{\prime \prime}(x)}{\left(u^{\prime}(x)\right)^{2}}+\sum_{s=1}^{N} \lambda_{s} \frac{\rho_{s}(x)}{\bar{\rho}_{s}(u(x))}=0
$$

satisfying boundary conditions $u(0)=0$ and $u(1)=1$.

We can deduce from this expression that the most probable density profile is given by $\boldsymbol{\rho}(x)=$ $\overline{\boldsymbol{\rho}}(x)$. The differential equation is indeed solved by the function $u(x)=x$ in this case because $\lambda_{1}+\cdots+\lambda_{N}=0$. Injecting in (73) makes the large deviation function vanish.

Remark. The thermodynamic equilibrium case can be of course recovered from the general case. Indeed we have $\overline{\boldsymbol{\rho}}(u)=\boldsymbol{\alpha}=\boldsymbol{\beta}=\boldsymbol{r}$ for all $u$. Moreover the differential equation (74) reduces to $u^{\prime \prime}(x)=0$ because $\lambda_{s}=0$ for all $s$ in this case. It is solved by the function $u(x)=x$. Injecting in (73) leads to (68) as expected.

Proof of (73). The proof presented here follows heavily the lines of the proof written in [20, 19] for the one species SSEP. For the sake of simplicity, we will present the proof for the case where $a+b=1$, but the generalisation to the other cases is straightforward.

We want to evaluate the probability $P_{L}\left(\left\{\boldsymbol{\rho}^{\{1\}}, \boldsymbol{\rho}^{\{2\}}, \ldots, \boldsymbol{\rho}^{\{n\}}\right\} \mid \boldsymbol{\alpha}, \boldsymbol{\beta}\right)$ to find $\rho_{s}^{\{k\}} \times l$ particles of species $s$ in the box $k$ for $s=1, \ldots, N$ and $k=1, \ldots, n$. This is done by summing the probabilities of all the configurations satisfying these constraints. For each of these configurations, we use the additivity principle (46) to divide the system into two part of size $L^{\prime}=k l$ (containing $k$ boxes) and $L^{\prime \prime}=(n-k) l$ (containing $n-k$ boxes), for a fixed $1 \leq k \leq n$. We thus obtain

$$
\begin{aligned}
P_{n l}\left(\left\{\boldsymbol{\rho}^{\{1\}}, \ldots, \boldsymbol{\rho}^{\{n\}}\right\} \mid \boldsymbol{\alpha}, \boldsymbol{\beta}\right)=-\frac{(k l) !((n-k) l) !}{(n l) !} \oint \frac{d u}{2 i \pi} \frac{1}{u^{k l+1}(1-u)^{(n-k) l+1}} \\
\quad \times P_{k l}\left(\left\{\boldsymbol{\rho}^{\{1\}}, \ldots, \boldsymbol{\rho}^{\{k\}}\right\} \mid \boldsymbol{\alpha}, \overline{\boldsymbol{\rho}}(u)\right) P_{(n-k) l}\left(\left\{\boldsymbol{\rho}^{\{k+1\}}, \ldots, \boldsymbol{\rho}^{\{n\}}\right\} \mid \overline{\boldsymbol{\rho}}(u), \boldsymbol{\beta}\right)
\end{aligned}
$$

In the large $l$ limit, evaluating the previous expression at the saddle point, we obtain the following equation for the large deviation function

$$
\begin{aligned}
& \mathcal{F}_{n}\left(\left\{\boldsymbol{\rho}^{\{1\}}, \ldots, \boldsymbol{\rho}^{\{n\}}\right\} \mid \boldsymbol{\alpha}, \boldsymbol{\beta}\right)=\max _{0<u<1} \frac{k}{n} \ln \left(\frac{n u}{k}\right)+\frac{n-k}{n} \ln \left(\frac{n(1-u)}{n-k}\right) \\
& +\frac{k}{n} \mathcal{F}_{k}\left(\left\{\boldsymbol{\rho}^{\{1\}}, \ldots, \boldsymbol{\rho}^{\{k\}}\right\} \mid \boldsymbol{\alpha}, \overline{\boldsymbol{\rho}}(u)\right)+\frac{n-k}{n} \mathcal{F}_{n-k}\left(\left\{\boldsymbol{\rho}^{\{k+1\}}, \ldots, \boldsymbol{\rho}^{\{n\}}\right\} \mid \overline{\boldsymbol{\rho}}(u), \boldsymbol{\beta}\right)
\end{aligned}
$$

We repeat $n$ times the same procedure to obtain

$$
\begin{array}{r}
\mathcal{F}_{n}\left(\left\{\boldsymbol{\rho}^{\{1\}}, \ldots, \boldsymbol{\rho}^{\{n\}}\right\} \mid \boldsymbol{\alpha}, \boldsymbol{\beta}\right)=\max _{0=u_{0}<u_{1}<\cdots<u_{n}=1} \frac{1}{n} \sum_{k=1}^{n} \mathcal{F}_{1}\left(\boldsymbol{\rho}^{\{k\}} \mid \overline{\boldsymbol{\rho}}\left(u_{k-1}\right), \overline{\boldsymbol{\rho}}\left(u_{k}\right)\right) \\
+\ln \left(\left(u_{k}-u_{k-1}\right) n\right)
\end{array}
$$

In the large $n$ limit, we can define the continuous variable $x=k / n$ and a function $u$ such that $u(x)=u_{k}$. The sequence $u_{k}$ being monotone, the difference $u_{k}-u_{k-1}$ is small in this limit. Hence 
we have that $\overline{\boldsymbol{\rho}}\left(u_{k-1}\right) \simeq \overline{\boldsymbol{\rho}}\left(u_{k}\right)$ and we can replace $\mathcal{F}_{1}\left(\boldsymbol{\rho}^{\{k\}} \mid \overline{\boldsymbol{\rho}}\left(u_{k-1}\right), \overline{\boldsymbol{\rho}}\left(u_{k}\right)\right)$ by the equilibrium value $\mathcal{F}_{1}\left(\boldsymbol{\rho}^{\{k\}} \mid \overline{\boldsymbol{\rho}}\left(u_{k}\right), \overline{\boldsymbol{\rho}}\left(u_{k}\right)\right)=B\left(\boldsymbol{\rho}^{\{k\}} \mid \overline{\boldsymbol{\rho}}\left(u_{k}\right)\right)$. We thus obtain

$$
\mathcal{F}(\{\boldsymbol{\rho}(x)\} \mid \boldsymbol{\alpha}, \boldsymbol{\beta})=\max _{u(x)} \int_{0}^{1} d x\left[B(\boldsymbol{\rho}(x), \overline{\boldsymbol{\rho}}(u(x)))+\ln u^{\prime}(x)\right],
$$

where the maximum is evaluated over the increasing functions $u$ satisfying $u(0)=0$ and $u(1)=1$. The Euler-Lagrange equation associated with the maximization over $u$ of this functional gives the differential equation (74).

Let us stress that exact computation, from finite size lattice, of the large deviation functional of the density profile has only be achieved on a few out-of-equilibrium models, including the SSEP [23, 24] and the ASEP [25, 26].

\section{Macroscopic fluctuation theory.}

\subsection{Hydrodynamic description of the multi-species SSEP.}

The macroscopic fluctuation theory (MFT) is a general approach that aims to describe out of equilibrium diffusive particle gases in the thermodynamic limit. It was developed a few years ago by Bertini, De Sole, Gabrielli, Jona-Lasinio and Landim [6, 7], and has proven to be an efficient way to compute fluctuations of the current and of the density profile. One strength of this theory is to describe the diffusive systems through only two key parameters, the diffusion constant $D(\rho)$ and the conductivity $\sigma(\rho)$ which depend on the local particle density $\rho$. These parameters can be determined case by case from the microscopic dynamics of the model. See [ 8 ] for a detailed review. Some validations from a microscopic point of view were realised for exactly solvable models including the SSEP [23, 24, 20, 19], and more recently a dissipative system [17]. All these works were related to systems with a single species of particles.

In this section we propose, based on the exact microscopic computations, a hydrodynamic description of the multi-species SSEP which extends the MFT to systems with several species of particles. We check the consistency with the exact results of the previous sections, the rigorous proof of the approach remains to be done (and lies beyond the scope of the present paper).

We define, when $L \rightarrow \infty$, the macroscopic density $\rho_{s}(x, t)$ of the species $s$ at time $t 4$ and at position $x \in[0,1]$ on the lattice by

$$
\rho_{s}(x, t) \simeq \frac{1}{2 \varepsilon L} \sum_{|i-L x| \leq L \varepsilon} \rho_{s}^{(i)},
$$

where $\varepsilon$ tends to zero and $L \varepsilon$ tends to infinity in the thermodynamic limit. We denote by $Q_{s}^{t,(i, i+1)}$ the algebraic number of particles of species $s$ that have crossed the bound between sites $i$ and $i+1$ during the time interval $[0, t]$. It allows us to consider

$$
Q_{s}(x, t) \simeq \frac{1}{2 \varepsilon L^{2}} \sum_{|i-L x| \leq L \varepsilon} Q_{s}^{t,(i, i+1)}
$$

\footnotetext{
${ }^{4}$ Note that in order to do this hydrodynamic limit, we have rescaled in all this section the time appearing in (4) with a factor $L^{2}$, as usual in this context.
} 
The macroscopic particle current $j_{s}(x, t)$ of species $s$ at time $t$ and at position $x$ is then defined as

$$
j_{s}(x, t)=\frac{\partial}{\partial t} Q_{s}(x, t) .
$$

\subsection{Rate function for the multi-species SSEP.}

The idea of the MFT is to express the probability to observe certain density profiles $\boldsymbol{\rho}(x, t)=$ $\left(\rho_{1}(x, t), \ldots, \rho_{N}(x, t)\right)$ and current profiles $\boldsymbol{j}(x, t)=\left(j_{1}(x, t), \ldots, j_{N}(x, t)\right)$ during the time interval $\left[t_{1}, t_{2}\right]$ as a large deviation principle. We present now one of the main result of this paper, which

gives a new perspective on the rate function of diffusive models with exclusion which can be seen as that of a model of free particles but with an additional constraint

$$
P(\{\boldsymbol{\rho}(x, t), \boldsymbol{j}(x, t)\}) \sim \exp \left[-L \int_{t_{1}}^{t_{2}} d t \int_{0}^{1} d x \sum_{s=1}^{N} \frac{\left(j_{s}(x, t)+\partial_{x} \rho_{s}(x, t)\right)^{2}}{4 \rho_{s}(x, t)}\right],
$$

where the fields satisfy the usual conservation law

$$
\frac{\partial}{\partial t} \boldsymbol{\rho}(x, t)=-\frac{\partial}{\partial x} \boldsymbol{j}(x, t)
$$

the boundary conditions

$$
\boldsymbol{\rho}(0, t)=\boldsymbol{\alpha}, \quad \boldsymbol{\rho}(1, t)=\boldsymbol{\beta}
$$

and the additional constraints

$$
\rho_{1}(x, t)+\cdots+\rho_{N}(x, t)=1, \quad j_{1}(x, t)+\cdots+j_{N}(x, t)=0 .
$$

The rate function (82) can be heuristically interpreted having in mind that, for Brownian particles, the diffusion constant is $D(\rho)=1$ and the conductivity is $\sigma(\rho)=2 \rho$. The functional (82) is exactly the one that describes a model of independent Brownian particles of $N$ different species, but on top of that we impose the exclusion constraint (85) which translates the fact that there is at most one particle per site. We recall that in our notation the holes (empty sites) are interpreted as a species of particles. This formula is supported by: (i) the consistency check with the large deviation functional of the density profile in the stationary state done in the next subsection, (ii) the following remark.

Remark. The well known case of the SSEP with a single species and holes can be recovered from (82) by setting $N=2$. Indeed, if we assume that species 1 plays the role of holes and species 2 the role of particles, we have in this case $j_{2}(x, t)=-j_{1}(x, t)=j(x, t)$ and $\rho_{2}(x, t)=1-\rho_{1}(x, t)=$ $\rho(x, t)$ due to the constraints (85). Then the rate function in (82) becomes

$$
\int_{t_{1}}^{t_{2}} d t \int_{0}^{1} d x \frac{\left(j(x, t)+\partial_{x} \rho(x, t)\right)^{2}}{4 \rho(x, t)(1-\rho(x, t))}
$$

which agrees with the known expression for the single species SSEP (recall that the diffusion constant is given by $D(\rho)=1$ and the conductivity by $\sigma(\rho)=2 \rho(1-\rho)$ ). 


\subsection{Check with the large deviation of the density profile.}

Following what was done in [20, 19, this framework allows us to express the probability to observe at time $\tau$ a density profile $\rho(x)$ in the stationary state. We have to identify how this deviation is produced, i.e. we have to find the optimal path $\boldsymbol{\rho}(x, t)$ such that $\boldsymbol{\rho}(x,-\infty)=\overline{\boldsymbol{\rho}}(x)$ and $\boldsymbol{\rho}(x, \tau)=\boldsymbol{\rho}(x)$ :

$$
\mathcal{F}(\{\boldsymbol{\rho}(x)\} \mid \boldsymbol{\alpha}, \boldsymbol{\beta})=\min _{\boldsymbol{\rho}(x, t), \boldsymbol{j}(x, t)} \int_{-\infty}^{\tau} d t \int_{0}^{1} d x \sum_{s=1}^{N} \frac{\left(j_{s}(x, t)+\partial_{x} \rho_{s}(x, t)\right)^{2}}{4 \rho_{s}(x, t)} .
$$

Note that the probability to observe a deviation in the density profile $\boldsymbol{\rho}(x)$ does not depend on the time on which this deviation occurs. It means that (87) does not depend on $\tau$.

$$
\mathcal{F}(\{\boldsymbol{\rho}(x)\} \mid \boldsymbol{\alpha}, \boldsymbol{\beta})=\min _{\delta \boldsymbol{\rho}(x), \boldsymbol{j}(x)}\left[\mathcal{F}(\{\boldsymbol{\rho}(x)-\delta \boldsymbol{\rho}(x)\} \mid \boldsymbol{\alpha}, \boldsymbol{\beta})+\delta \tau \int_{0}^{1} d x \sum_{s=1}^{N} \frac{\left(j_{s}(x)+\rho_{s}^{\prime}(x)\right)^{2}}{4 \rho_{s}(x)}\right],
$$

where we have used the definitions $\boldsymbol{\rho}(x)-\delta \boldsymbol{\rho}(x)=\boldsymbol{\rho}(x, \tau-\delta \tau)$ and $\boldsymbol{j}(x)=\boldsymbol{j}(x, \tau)$. The conservation law reads $\delta \boldsymbol{\rho}(x)=-\boldsymbol{j}^{\prime}(x) \times \delta \tau$. If we define

$$
U_{s}(x)=\frac{\delta \mathcal{F}(\{\boldsymbol{\rho}(x)\} \mid \boldsymbol{\alpha}, \boldsymbol{\beta})}{\delta \rho_{s}(x)}
$$

we can write using (88) an equation satisfied by the $U_{s}(x)$ 's. Indeed, maximising (88) over the current profile $\boldsymbol{j}(x)$ with the constraint (85) yields

$$
j_{s}(x)=-\rho_{s}^{\prime}(x)+2 \rho_{s}(x) U_{s}^{\prime}(x)-2 \rho_{s}(x) \mu(x),
$$

with the Lagrange multiplier

$$
\mu(x)=\sum_{s=1}^{N} \rho_{s}(x) U_{s}^{\prime}(x) .
$$

Using the fact that $\sum_{s=1}^{N} j_{s}(0) U_{s}(0)=\sum_{s=1}^{N} j_{s}(1) U_{s}(1)=0$ (because of the boundary conditions (84)), we can perform an integration by part and derive an equation satisfied by the functions $U_{s}^{\prime}(x)$

$$
\int_{0}^{1} d x\left[\sum_{s=1}^{N}\left(\rho_{s}^{\prime}(x) U_{s}^{\prime}(x)-\rho_{s}(x) U_{s}^{\prime}(x)^{2}\right)+\left(\sum_{s=1}^{N} \rho_{s}(x) U_{s}^{\prime}(x)\right)^{2}\right]=0 .
$$

We can check that the large deviation functional exactly computed in section 5 indeed fulfills this equation. We deduce from (73) that

$$
U_{s}(x)=\ln \left(\frac{\rho_{s}(x)}{\bar{\rho}_{s}(u(x))}\right)+1,
$$

where the function $u$ satisfies (74). Using the constraints (85) and the expression of $U_{s}(x)$, the differential equation (74) can be rewritten

$$
\frac{u^{\prime \prime}(x)}{u^{\prime}(x)}=-\sum_{s=1}^{N} \rho_{s}(x) U_{s}^{\prime}(x)
$$


This permits to show that

$$
\left(\frac{u^{\prime \prime}}{u^{\prime}}\right)^{\prime}(x)=\sum_{s=1}^{N}\left(\rho_{s}^{\prime}(x) U_{s}^{\prime}(x)-\rho_{s}(x) U_{s}^{\prime}(x)^{2}\right)+\left(\sum_{s=1}^{N} \rho_{s}(x) U_{s}^{\prime}(x)\right)^{2} .
$$

Then we deduce that the left hand side of (92) is equal to

$$
\int_{0}^{1} d x\left(\frac{u^{\prime \prime}}{u^{\prime}}\right)^{\prime}(x)=\frac{u^{\prime \prime}(1)}{u^{\prime}(1)}-\frac{u^{\prime \prime}(0)}{u^{\prime}(0)}=0
$$

because $u^{\prime \prime}(1)=u^{\prime \prime}(0)=0$ thanks to (74).

Let us stress that the Hamilton-Jacobi equation (92) obtained when trying to compute the large deviation functional of the density profile from the MFT formalism has only been solved in a few models including the weakly asymmetric simple exclusion process [27, 18] and the KipnisMarchioro-Presutti model [30, 9].

\section{Integrability.}

\subsection{Integrability in a nutshell.}

In this section we summarize the integrability framework in which the multi-species SSEP with boundaries takes part. As already mentioned, the Markov matrix (5) that governs the stochastic dynamics of the model is integrable. It means that the Markov matrix $M$ belongs to a set of commuting operators which are all encompassed in a generating function $t(x)$ called the transfer matrix. The commutation between the operators is ensured by the key property

$$
[t(x), t(y)]=0 .
$$

For systems with open boundaries [39], the transfer matrix is build from two fundamental blocks, the $R$-matrix and the $K$-matrices. We present them in the case of the multi-species SSEP.

The $R$-matrix $\check{R}(x)=(x P+1) /(x+1)$ acting in $\mathbb{C}^{N} \otimes \mathbb{C}^{N}$, where $P$ is the permutation operator defined right after (10), satisfies the braided Yang-Baxter equation

$$
(\check{R}(x) \otimes 1)(1 \otimes \check{R}(x+y))(\check{R}(y) \otimes 1)=(1 \otimes \check{R}(y))(\check{R}(x+y) \otimes 1)(1 \otimes \check{R}(x)) .
$$

This equation holds in $\mathbb{C}^{N} \otimes \mathbb{C}^{N} \otimes \mathbb{C}^{N}$. The local jump operator $m$ defined in (10) is intimately linked to $\check{R}$ through $m=\check{R}^{\prime}(0)$.

The $K$ matrices $K(x)=1+2 x a B /(x+a)$ for the left boundary and $\bar{K}(x)=1+2 x b \bar{B} /(x-b)$ for the right boundary, where $B$ and $\bar{B}$ are the boundary matrices defined in (77) and (9), both satisfy the braided reflection equation

$$
\check{R}(x-y)(K(x) \otimes 1) \check{R}(x+y)(K(y) \otimes 1)=(K(y) \otimes 1) \check{R}(x+y)(K(x) \otimes 1) \check{R}(x-y)
$$

This equation holds in $\mathbb{C}^{N} \otimes \mathbb{C}^{N}$. The boundary matrices $B$ and $\bar{B}$ are obtained through $B=$ $K^{\prime}(0) / 2$ and $\bar{B}=-\bar{K}^{\prime}(0) / 2$.

These objects allow us to construct the double row transfer matrix [39]

$$
t(x)=\operatorname{tr}_{0}\left(\widetilde{K}_{0}(x) R_{0, L}(x) \ldots R_{0,1}(x) K_{0}(x) R_{1,0}(x) \ldots R_{L, 0}(x)\right)
$$


where $R(x)=P . \check{R}(x)$ and $\widetilde{K}(x)=\operatorname{tr}_{0}\left(\bar{K}_{0}(-x)\left(\left(R_{01}(2 x)^{t_{1}}\right)^{-1}\right)^{t_{1}} P_{01}\right)$. Like the Markov matrix, the transfer matrix acts in $\underbrace{\mathbb{C}^{N} \otimes \cdots \otimes \mathbb{C}^{N}}_{L}$. The indices in (100) indicate the copies of $\mathbb{C}^{N}$ in the tensor space in which the matrices are acting. Remark that we used an additional copy of $\mathbb{C}^{N}$ with number 0 which is traced out.

The Markov matrix $M$ is then simply given by $M=t^{\prime}(1) / 2$. The commutation property of the transfer matrix (97) is ensured by the Yang-Baxter equation (98) and the reflection equation (99). The proof can be found in [39]. A more detailed review of the integrable formalism for open exclusion processes can be found in [16].

\subsection{More integrable boundaries.}

As already mentioned, the boundary conditions of the model studied in this paper are specific solutions of the reflection equation (99). These solutions were indeed of particular interest because of their very simple physical interpretation. Nevertheless there exist several other solutions to (99) which provide other integrable stochastic boundary matrices $B$ and $\bar{B}$. The solutions of the reflection equation (99) has been classified in [35. We present here, without proof, classes of stochastic boundaries among this classification. We divide the $N$ species into $p$ distinct families $F_{1}, \ldots, F_{p}$ of non-vanishing cardinalities $f_{1}, \ldots, f_{p}$ at the left boundary and into $q$ distinct families $G_{1}, \ldots, G_{q}$ of non-vanishing cardinalities $g_{1}, \ldots, g_{q}$ at the right boundary. We hence have two different partitions $\{1, \ldots, N\}=\bigsqcup_{k=1}^{p} F_{k}=\bigsqcup_{k=1}^{q} G_{k}$. We define $2 N$ non negative numbers $\alpha_{1}, \ldots, \alpha_{N}$ for the left boundary and $\beta_{1}, \ldots, \beta_{N}$ for the right boundary with the constraints

$$
\text { for all } 1 \leq k \leq p, \quad \sum_{s \in F_{k}} \alpha_{s}=1,
$$

and

$$
\text { for all } 1 \leq k \leq q, \quad \sum_{s \in G_{k}} \beta_{s}=1 .
$$

The left boundary conditions are given by

$$
B\left|s^{\prime}\right\rangle=-\frac{1}{a}\left|s^{\prime}\right\rangle+\sum_{s \in F_{k}} \frac{\alpha_{s}}{a}|s\rangle, \quad 1 \leq s^{\prime} \leq N .
$$

$k$ in (103) is such that $s^{\prime} \in F_{k}$. Remark that in the particular case where the family of $s^{\prime}$ contains only one species, i.e. $F_{k}=\left\{s^{\prime}\right\}$, we get from the constraints that $\alpha_{s^{\prime}}=1$ and hence $B\left|s^{\prime}\right\rangle=0$.

In the same way, the right boundary conditions are given by

$$
\bar{B}\left|s^{\prime}\right\rangle=-\frac{1}{b}\left|s^{\prime}\right\rangle+\sum_{s \in G_{k}} \frac{\beta_{s}}{b}|s\rangle, \quad 1 \leq s^{\prime} \leq N,
$$

with $k$ such that $s^{\prime} \in G_{k}$. Note that when we have a single family on the left and a single family on the right, i.e. when $p=q=1$, then the boundary conditions reduce to the one studied in details in this paper. To illustrate these boundary conditions, we give some examples in the case 
$N=4$ for the left boundary:

$$
\begin{aligned}
& B=\frac{1}{a}\left(\begin{array}{cccc}
\alpha_{1}-1 & \alpha_{1} & 0 & \alpha_{1} \\
\alpha_{2} & \alpha_{2}-1 & 0 & \alpha_{2} \\
0 & 0 & 0 & 0 \\
\alpha_{4} & \alpha_{4} & 0 & \alpha_{4}-1
\end{array}\right), \quad \text { with } \quad \alpha_{1}+\alpha_{2}+\alpha_{4}=1 \\
& B=\frac{1}{a}\left(\begin{array}{cccc}
\alpha_{1}-1 & \alpha_{1} & 0 & 0 \\
\alpha_{2} & \alpha_{2}-1 & 0 & 0 \\
0 & 0 & \alpha_{3}-1 & \alpha_{3} \\
0 & 0 & \alpha_{4} & \alpha_{4}-1
\end{array}\right) \text {, with } \quad \alpha_{1}+\alpha_{2}=1 \text { and } \alpha_{3}+\alpha_{4}=1,(106) \\
& B=\frac{1}{a}\left(\begin{array}{cccc}
\alpha_{1}-1 & 0 & \alpha_{1} & 0 \\
0 & \alpha_{2}-1 & 0 & \alpha_{2} \\
\alpha_{3} & 0 & \alpha_{3}-1 & 0 \\
0 & \alpha_{4} & 0 & \alpha_{4}-1
\end{array}\right) \text {, with } \quad \alpha_{1}+\alpha_{3}=1 \text { and } \alpha_{2}+\alpha_{4}=1,(107) \\
& B=\frac{1}{a}\left(\begin{array}{cccc}
0 & 0 & 0 & 0 \\
0 & \alpha_{2}-1 & \alpha_{2} & 0 \\
0 & \alpha_{3} & \alpha_{3}-1 & 0 \\
0 & 0 & 0 & 0
\end{array}\right) \text {, with } \quad \alpha_{2}+\alpha_{3}=1
\end{aligned}
$$

Examples of right boundaries in the case $N=4$ are obtained by replacing $\alpha_{i}$ by $\beta_{i}$ and $a$ by $b$ in the matrices (105)-(108) above.

\section{Conclusion}

In this work we have introduced a multi-species generalization of the SSEP with open boundaries. The boundary conditions are carefully tuned to maintain the integrability of the model and are physically interpreted as the coupling with particles reservoirs with fixed densities of each species. We have expressed analytically the steady state using the matrix ansatz technique and it turns out that the matrices involved belong to a simple Lie algebra. Using this convenient algebraic structure we derived exact expressions of physical quantities including the large deviation functional of the density profile. We proposed a description of the model in the framework of the MFT and check the consistency with the exact computations done on the finite size lattice.

There is of course a lot of work that remains to be done on this model. One thing that is of prime interest in the context of out-of-equilibrium statistical physics is to study the fluctuations of the particles currents. The usual way to do that is to perform a current counting deformation of the Markov matrix. It would be of interest to extend to this multi-species model the results on the current fluctuations obtained for the one species open SSEP [21, 19]. We could also directly compute the fluctuations of the current from the MFT formalism, assuming that the density profile which produces an atypical current is time independent. In particular we could try to see if the additivity formalism developed in [11] can be extended to a model with several species of particles. The results should be compared with the one given by the current counting deformation of the Markov matrix.

It could be also interesting to study in detail other combinations of integrable boundaries given in section 7. In particular, following the general approach of the matrix ansatz for integrable systems developed in [37] and [16], it should be possible to construct the non-equilibrium 
stationary state in a matrix product form. It may be enlightening to see how the matrix product solution is modified by the change of boundary conditions and whether the additivity principle holds. A description in the context of the MFT of such models also remains to be done.

\section{Acknowledgments}

We are grateful to Caley Finn for helpful discussions. We would like to warmly thank Nicolas Crampé, Martin Evans, Kirone Mallick and Eric Ragoucy for their interest and suggestions.

\section{References}

[1] C. Arita, Remarks on the multi-species exclusion process with reflective boundaries, J. Phys. A 45 (2012) 155001 and arXiv:1112.5585.

[2] C. Arita, A. Ayyer, K. Mallick and S. Prolhac, Recursive structures in the multi-species TASEP, J. Phys. A 44 (2011) 335004 and arXiv:1104.3752.

[3] C. Arita, A. Ayyer, K. Mallick and S. Prolhac, Generalized matrix Ansatz in the multispecies exclusion process - the partially asymmetric case, J. Phys. A 45 (2012) 195001 and arXiv: 1201.0388.

[4] A. Ayyer, J.L. Lebowitz and E.R. Speer, On the Two Species Asymmetric Exclusion Process with Semi-Permeable Boundaries, J. Stat. Phys. 135 (2009) 1009 and arXiv:0807.2423.

[5] S. Belliard and N. Crampe, Heisenberg XXX Model with General Boundaries: Eigenvectors from Algebraic Bethe Ansatz, SIGMA 9 (2013) 072 and arXiv:1309.6165.

[6] L. Bertini, A. De Sole, D. Gabrielli, G. Jona-Lasinio and C.Landim, Fluctuations in stationary non equilibrium states of irreversible processes, Phys. Rev. Lett. 87 (2001) 040601 and arXiv: cond-mat/0104153.

[7] L. Bertini, A. De Sole, D. Gabrielli, G. Jona-Lasinio and C. Landim, Macroscopic Fluctuation Theory for stationary non-equilibrium states, J. Stat. Phys. 107 (2002) 635 and arXiv: cond-mat/0108040.

[8] L. Bertini, A. De Sole, D. Gabrielli, G. Jona-Lasinio and C. Landim, Macroscopic Fluctuation Theory, Rev. Mod. Phys. 87 (2015) 593 and arXiv:1404.6466.

[9] L. Bertini, D. Gabrielli and J. Lebowitz, Large deviation for a stochastic model of heat flow, J. Stat. Phys. 121 (2005) 843 and arXiv: cond-mat/0501681.

[10] R. A. Blythe and M.R. Evans, Nonequilibrium steady states of matrix-product form: a solver's guide, J. Phys. A 40 (2007) R333 and arXiv:0706.1678.

[11] T. Bodineau and B. Derrida, Current fluctuations in nonequilibrium diffusive systems: an additivity principle, Phys. Rev. Lett. 92 (2004) 180601 and arXiv:cond-mat/0402305.

[12] L. Cantini, J. de Gier and M. Wheeler, Matrix product formula for Macdonald polynomials, J. Phys. A 48 (2015) 384001 and arXiv:1505.00287. 
[13] T. Chou, K. Mallick and R. K. P. Zia, Non-equilibrium statistical mechanics: From a paradigmatic model to biological transport, Rep. Prog. Phys. 74 (2011) 116601 and arXiv: 1110.1783.

[14] N. Crampe, M.R. Evans, K. Mallick, E. Ragoucy, M. Vanicat, Matrix product solution to a 2-species TASEP with open integrable boundaries, to appear in J. Phys. A and arXiv: 1606.08148.

[15] N. Crampe, C. Finn, E. Ragoucy and M. Vanicat, Integrable boundary conditions for multispecies ASEP, J. Phys. A 49 (2016) 375201 and arXiv:1606.01018.

[16] N. Crampe, E. Ragoucy and M. Vanicat, Integrable approach to simple exclusion processes with boundaries. Review and progress, J. Stat. Mech. (2014) P11032 and arXiv:1408.5357.

[17] N. Crampe, E. Ragoucy, V. Rittenberg and M. Vanicat, Integrable dissipative exclusion process: Correlation functions and physical properties, Phys. Rev. E and arXiv:1603.06796.

[18] A. Demasi, E. Presutti and E. Scacciatelli, The weakly asymmetric simple exclusion process, Ann. Inst. Henri Poincar-Probab. Stat. 25 (1989) 1.

[19] B. Derrida, Non-equilibrium steady states: fluctuations and large deviations of the density and of the current, J. Stat. Mech. (2007) P07023 and arXiv:cond-mat/0703762.

[20] B. Derrida, Microscopic versus macroscopic approaches to non-equilibrium systems, J. Stat. Mech. (2011) P01030 and arXiv:1012.1136.

[21] B. Derrida, B. Doucot, P.-E. Roche, Current fluctuations in the one dimensional Symmetric Exclusion Process with open boundaries, J. Stat. Phys. 115 (2004) 717 and arXiv: cond-mat/0310453.

[22] B. Derrida, M.R. Evans, V. Hakim and V. Pasquier, Exact solution of a 1d asymmetric exclusion model using a matrix formulation, J. Phys. A 26 (1993) 1493.

[23] B. Derrida, J. Lebowitz and E. Speer, Free energy functional for nonequilibrium systems: an exactly solvable case, Phys. Rev. Lett. 87 (2001) 150601 and arXiv:cond-mat/0105110.

[24] B. Derrida, J. Lebowitz and E. Speer, Large deviation of the density profile in the steady state of the open symmetric simple exclusion process, J. Stat. Phys. 107 (2002) 599 and arXiv: cond-mat/0109346.

[25] B. Derrida, J. Lebowitz and E. Speer, Exact free energy functional for a driven diffusive open stationary nonequilibrium system, Phys. Rev. Lett. 89 (2002) 030601 and arXiv: cond-mat/0203161.

[26] B. Derrida, J. Lebowitz and E. Speer, Exact large deviation functional of a stationary open driven diffusive system: the asymmetric exclusion process, J. Stat. Phys. 110 (2003) 775 and arXiv: cond-mat/0205353.

[27] C. Enaud and B. Derrida, Large deviation functional of the weakly asymmetric exclusion process, J. Stat. Phys. 114 (2004) 537 and arXiv:cond-mat/0307023. 
[28] M.R. Evans, P.A. Ferrari and K. Mallick, Matrix Representation of the Stationary Measure for the multi-species TASEP, J. Stat. Phys. 135 (2009) 217 and arXiv:0807.0327.

[29] V. Karimipour, Multispecies asymmetric simple exclusion process and its relation to traffic flow, Phys. Rev. E 59 (1999) 205 and arXiv:cond-mat/9808220.

[30] C. Kipnis, C. Marchioro and E. Presutti, Heat-flow in an exactly solvable model, J. Stat. Phys. 27 (1982) 65.

[31] J. Krug, Boundary-induced phase transitions in driven diffusive systems, Phys. Rev. Lett. 67 (1991) 1882.

[32] A. Kuniba, S. Maruyama and M. Okado, Multispecies TASEP and the tetrahedron equation, J. Phys. A 49 (2016) 114001 and arXiv:1509.09018; Multispecies TASEP and combinatorial $R$, J. Phys. A 48 (2015) 34FT02 and arXiv:1506.04490.

[33] K. Mallick, S. Mallick and N. Rajewsky, Exact solution of an exclusion process with three classes of particles and vacancies, J. Phys. A 32 (1999) 8399 and arXiv:cond-mat/9903248.

[34] C.S. Melo, G.A.P. Ribeiro and M.J. Martins Bethe ansatz for the XXX-S chain with nondiagonal open boundaries, Nucl. Phys. B 711 (2005) 565 and arXiv:nlin/0411038.

[35] M. Mintchev, E. Ragoucy and P. Sorba, Spontaneous symmetry breaking in the gl(N)-NLS hierarchy on the half line, J. Phys. A 34 (2001) 8345 and arXiv:hep-th/0104079.

[36] S. Prolhac, M.R. Evans and K. Mallick, Matrix product solution of the multi-species partially asymmetric exclusion process, J. Phys. A 42 (2009) 165004 and arXiv:0812.3293.

[37] T. Sasamoto and M. Wadati, Stationary states of integrable systems in matrix product form, J. Phys. Soc. Japan 66 (1997) 2618.

[38] G. Schütz and E. Domany, Phase transitions in an exactly soluble one-dimensional exclusion process, J. Stat. Phys. 72 (1993) 277.

[39] E. K. Sklyanin, Boundary conditions for integrable quantum systems, J. Phys. A 21 (1988) 2375 .

[40] M. Uchiyama, Two-Species Asymmetric Simple Exclusion Process with Open Boundaries, Chaos, Solitons \& Fractals 35 (2008) 398 and arXiv: cond-mat/0703660. 